\title{
INERTIAL FRAME DETERMINATION FROM HIPPARCOS OBSERVATIONS OF MINOR PLANETS .
}

\author{
D. HESTROFFER \\ Bureau des longitudes \\ 77 Avenue Denfert Rochereau \\ 75014 Paris \\ France
}

\begin{abstract}
Observations of 48 minor planets by Hipparcos should lead to a good determination of the inertial reference frame. Analysis of one year observations showed periodic effects in the o-c due to a rotation between the Hipparcos system and the inertial system. By extending this result to a 2.5 years simulation and by studying the impact of added ground-based observations, it appears that the orbital elements of the Earth and the parameters of a rotation of the system can be obtained with an accuracy better than 5 mas.
\end{abstract}

\section{INTRODUCTION}

The Hipparcos satellite launched in august 1989 provides positions for 48 minor planets. Its scanning law is based on a regular precession of its spin axis around the sun (with a revolving angle of $43^{\circ}$ ) wich cause the observation of a planet to be around quadrature only (Morando and Lindegren 1982). A single observation gives the projected position of a planet on a reference great circle (RGC, it is a mean circle of about 5 satellite revolutions), no significant information on the other coordinate of the object is available.

After studying the data obtained for one year, we made a simulation for a 2.5 years period (November 89 - May 92) of the observations in order to get a first approximation of the inertial frame determination precision that occurs in this mission. In this simulation no occultation by the Earth or Moon was taken into account. The condition for observing a minor planet was, first, that the planet be near quadrature and, second, that it crosses one of the two telescope fields $\left(0^{\circ} 9 \times 0^{\circ} 9\right)$ during one satellite revolution with a magnitude lower than 12.5 mag.

\section{THE CONDITION EQUATIONS}

Let $\mathbf{z}$ be the unit vector defining a RGC axis, $\mathbf{z}$ is deduced from the scanning law and is taken as being the satellite spin axis position every 0.4 days. Let $\mathbf{u}$ (resp. $\mathbf{u}^{\prime}$ ) be the geocentric (resp. satellicen- 
tric) vector towards the apparent planet position, an observation by Hipparcos yields the difference between the observed and calculated position :

$$
\mathrm{O}-\mathrm{C}=\frac{\mathbf{z} \times \mathbf{u}^{\prime}}{\left(\mathbf{z} \times \mathbf{u}^{\prime}\right)^{2}} \cdot \Delta \mathbf{u}^{\prime} \approx \frac{\mathbf{z} \times \mathbf{u}}{(\mathbf{z} \times \mathbf{u})^{2}} \cdot \Delta \mathbf{u}
$$

where $\Delta \mathbf{u}$ is the correction to the computed position expressed in terms of the Earth and planet orbit corrections and an orientation correction of the Hipparcos system. The unknowns are, for the Earth and planet orbit corrections those advocated by Brouwer and Clemence (1961) :

$$
\begin{aligned}
& \left(\Delta r^{\prime}+\Delta l_{0}^{\prime} ; \Delta p^{\prime} ; \Delta q^{\prime} ; e^{\prime} \Delta r^{\prime} ; \Delta e^{\prime}\right) \quad \text { or } \mathbf{d q} q_{0} \text { for the Earth } \\
& \left(\Delta r+\Delta l_{0} ; \Delta p ; \Delta q ; e \Delta r ; \Delta e ; \frac{\Delta a}{a}\right) \text { or } \mathbf{d} q_{i} \text { for the planet } i
\end{aligned}
$$

where $e$ is the eccentricity, $a$ is the semi-major axis, $l_{0}$ is the mean anomaly, $\Delta p, \Delta q$ and $\Delta r$ are infinitesimal rotations around axis toward respectively perihelion, $90^{\circ}+$ perihelion and the orbital pole.

To these we have added three more unknowns for the system rigid rotation :

$$
\left(w_{x}, w_{y}, w_{z}\right)=w
$$

The input parameters are : the minor planets osculating elements given by Bec-Borsenberger (1990), the Earth elements taken from Bretagnon's VSOP82 theory (Bretagnon 1982), the Earth and minor planets ephemerides of the Bureau des longitudes.

\section{THE NORMAL EQUATIONS AND SOLUTION}

We have adopted the mean square method of resolution described by Lindegren and Söderhjelm (1982), we can write for each planet $i$ the system of $n$ equations :

$$
A_{i} \cdot v+B_{i} \cdot d q_{i}=(0-c)_{i}
$$

where $n$ is the number of observations for this planet and $\mathbf{v}$ is the $(8,1)$ vector corresponding to the unknowns $d q_{0}$ and $\mathbf{w}$. The advantage of this method is that $\mathbf{v}$ and the dqi's can be calculated in separate steps and that only inversions of matrix of size $(8,8)$ and $(6,6)$ respectively are needed. For the vector o-c we assumed, first, that the phase effects were negligeable and, second, that it is only due to an initial constant rotation $\mathbf{w}$ (orientation error) between 
the Hipparcos system and the dynamical frame. Such a rotation yields the difference for each observation :

$$
\Delta=\frac{\mathbf{z} \times \mathbf{u}^{\prime}}{\left(\mathbf{z} \times \mathbf{u}^{\prime}\right)^{2}} \cdot\left(\mathbf{u}^{\prime} \times \mathbf{w}\right) \approx-\mathbf{z} \cdot \mathbf{w}
$$

Figure 1 shows the smoothed o-c obtained after one year of observation and the function - z.w. Smoothing this data diminishes the observation noise and absorbs the residuals due to the error in the orbital elements of each planet. Thus the regular precession of the satellite spin axis will make this difference periodical with periods of about 57 days and one year. We take o-c following this periodical part with an observational gaussian noise :

$$
0-c=-z \cdot w+g
$$

where $\mathbf{w}=(19.4 ;-51.1 ;-112)$ in mas, and $g \in N\left(0 ; \sigma^{2}\right)$

the mean error of one observation $\sigma$ is taken as a function of the magnitude only :

$$
\sigma=1.5 / \sqrt{2} \cdot \sqrt{4+10(0.52 \cdot H-4.45)} \text { in mas }
$$

this empirical function gives a first estimation of the mean error obtained after reduction of the observed positions for two successive crossings of the telescope fields.

As a consequence of the observations geometry, the orientation parameters for the Earth orbit and the system orientation cannot be well determined. For a better separation of the parameters we used the following linear combinations of the unknowns :

$$
\mathrm{w}_{\mathrm{x}}-\Delta \mathrm{p}^{\prime}-\Delta \mathrm{p} \quad ; \quad \mathrm{w}_{\mathrm{y}}-\Delta \mathrm{q}^{\prime}-\Delta \mathrm{q} \quad ; \quad \mathrm{w}_{\mathrm{z}}-\left(\Delta \mathrm{r}^{\prime}+\Delta \mathrm{l}_{\mathrm{o}}\right)-\left(\Delta \mathrm{r}+\Delta \mathrm{l}_{\mathrm{o}}\right)
$$

These combinations are coherent with the method of resolution used because the $\mathbf{d q} \mathbf{q}_{i}$ 's are assumed to be all equal to the null vector.

Table 1 shows the results obtained after a classical least square method was applied to solve the weighted equations (the weight is $\left.1 / \sigma^{2}\right)$. The plane of the ecliptic is determined to within 1 mas while the parameters corresponding to the orientation in this plane have the lowest precisions. This is due partly to the fact that observations occur near quadrature, thus the adjunction of groundbased observations around opposition could help diminishing the mean errors obtained for $\Delta r^{\prime}+\Delta l^{\prime} \circ$ and $w_{z}$. 
Table 1 : Solution for a 2.5 years simulation.

\begin{tabular}{|cr|c|}
\multicolumn{2}{c}{ System rotation } & \multicolumn{2}{c}{ (mas) } & mean error \\
\hline$w_{x}-\Delta p^{\prime}-\Delta p$ & 19.11 & 0.95 \\
\hline$w_{y}-\Delta q^{\prime}-\Delta q$ & -51.36 & 0.95 \\
\hline$w_{z}-\Delta r^{\prime}-\Delta l^{\prime} \circ$ & -113.85 & 3.99 \\
$-\Delta r-\Delta l_{0}$ & & \\
\hline
\end{tabular}

\begin{tabular}{|c|c|c|}
\hline \multicolumn{2}{|c|}{ Earth correction (mas) } & mean error \\
\hline$\Delta r^{\prime}+\Delta l_{0}^{\prime}$ & 1.95 & 3.92 \\
\hline$\Delta p^{\prime}$ & 0.64 & 0.93 \\
\hline$\Delta q^{\prime}$ & 0.89 & 1.00 \\
\hline$e^{\prime} \Delta r^{\prime}$ & -0.12 & 1.85 \\
\hline$\Delta \mathrm{e}^{\prime}$ & 1.43 & 1.70 \\
\hline
\end{tabular}

+ : SMOOTHED O-C

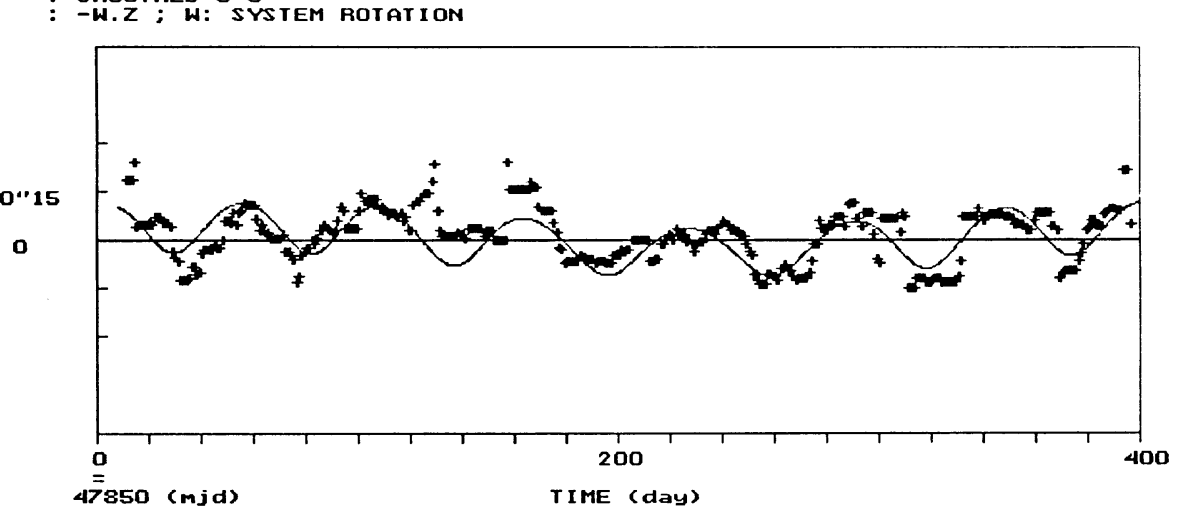

Figure 1 : Smoothed o-c

\section{ADJUNCTION OF GROUND-BASED OBSERVATIONS}

We suppose that the planets and the Earth have all circular orbits in the plane of the ecliptic and we restrict the unknowns to the corrections to the mean longitude of the Earth $\left(\Delta \mathrm{L}^{\prime} \mathrm{o}\right)$ and of a planet $\left(\Delta \mathrm{L}_{0}\right)$.

We can write from Numerov (1935) the equation of condition :

$$
(\gamma+1 / \gamma-2 \cos \psi) . \Delta \alpha=(\gamma-\cos \psi) \cdot \Delta \mathrm{L}_{0}+(1 / \gamma-\cos \psi) . \Delta \mathrm{L}_{\mathrm{o}}^{\prime}
$$


with (see fig 2):

$\left(r^{\prime}, I^{\prime}\right)$ and $(r, l)$ the Earth and planet heliocentric polar coordinates, $\gamma=r / r^{\prime}$,

$\psi=1 '-1$,

$\Delta \alpha$ the difference of longitude between the observed and calculated position.

For the Hipparcos observations, the equation of condition refers to a RGC which makes an angle $\beta \in\left[47^{\circ} ; 133^{\circ}\right]$ with the ecliptic. The difference between the observed and calculated position is :

$$
0-c=\cos \beta \cdot \Delta \alpha
$$

where $\cos \beta$ is also a function of $\psi$ and $\gamma$.

Assuming the same density $d$ of uniformly spaced observations for all the planets, we can write the mean error for $\Delta \mathrm{L}_{0}^{\prime}$ :

$$
\sigma\left(\Delta L^{\prime}{ }_{0}\right)=\sigma_{0} / \sqrt{d} \cdot \eta ; \sigma_{0} \text { is the mean error per unit weight }
$$

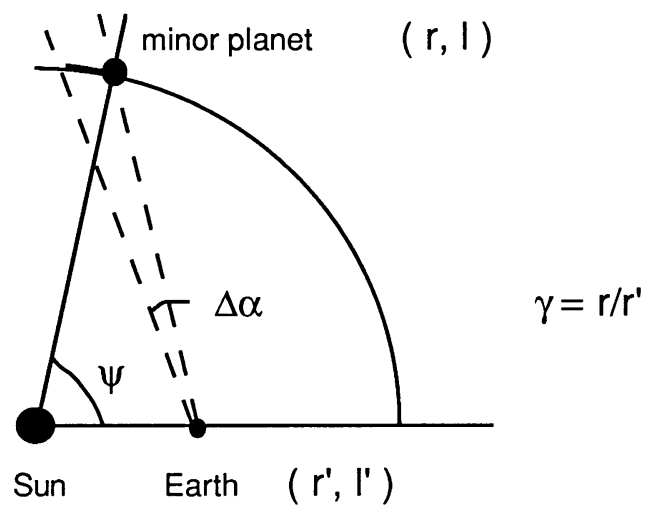

Figure 2: Difference of longitude $\Delta \alpha$

Let the Hipparcos observations be made over the arcs $\left[\psi_{1} ; \psi_{2}\right]$ and $\left[-\psi_{2} ;-\psi_{1}\right]$ around quadrature, while ground-based observations near opposition take place on the arc $\left[-\psi_{3} ; \psi_{3}\right]$. The angles $\psi_{1}$ and $\psi_{2}$ are functions of $\gamma$ and we take $\psi_{3}=35^{\circ}$. The equations corresponding to ground-based observations were weighted by a factor 0.01 (it corresponds to an error of 100 mas if the mean error of an Hipparcos observation is 10 mas).

We obtained values for $\eta$ (the precision factor for $\Delta L^{\prime}$ o) when considering two cases; the first case uses only observations from the satellite $(\eta=1.03)$, and the second case includes observations 
around opposition $(\eta=0.96)$. We can see that the adjunction of ground-based observations gives an amelioration of about $7 \%$ on the determination of $\Delta \mathrm{L}^{\prime} \mathrm{o}$.

\section{CONCLUSION}

We have shown that the orientation of the dynamical ecliptic could be tied to the Hipparcos system with a precision better than 5 mas assuming that no systematic phase and scattering effects would biased the results; and that the consideration of ground-based observations around opposition could be fruitfull for the separation of the mean longitude of the Earth and the planets and consequently for the determination of the orientation parameters in the ecliptical plane.

\section{ACKNOWLEDGEMENTS}

My warmest thanks are due to Dr. B. Morando for assistance and stimulating conversations.

\section{REFERENCES}

Bec-Borsenberger A. : 1990 Astron. Astrophys. Sup. Ser. 86, 299-349 Bretagnon P. : 1982 Astron. Astrophys. 114, 278-288

Morando B., Lindegren L. : 1989 ESA SP-1111, 3, 269-281

Numerov B.V. : 1936 Astron. J 45, 105-111

Söderhjelm S., Lindegren L. : 1982 Astron. Astrophys. 110,156-162 